\title{
Um aplicativo para estimulação da memória visual em crianças disléxicas por meio do jogo de palavras cruzadas
}

\author{
Guilherme Carvalho Lucena $^{1}$, Luciana Cidrim ${ }^{2}$, Francisco Madeiro ${ }^{1}$ \\ ${ }^{1}$ Centro de ciências e tecnologia - Universidade Católica de Pernambuco (UNICAP) \\ Recife - PE - Brasil \\ ${ }^{2}$ Programa de Pós-Graduação em Ciências da Linguagem - Universidade Católica de \\ Pernambuco (UNICAP) \\ Recife - PE - Brasil \\ lucena9682@gmail.com, lucianacidrimeunicap.br, \\ madeiro@dei.unicap.br
}

\begin{abstract}
This paper presents a crossword game application for smartphone devices, which aims to stimulate the visual memory of words with phonological awareness of dyslexic children. The application also aims to collect user data in order to generate application usage statistics, and can report, for example, the higher error frequency words. Information obtained through the application of questionnaires for speech-language pathologists points out the originality and adequacy of the application.
\end{abstract}

Resumo. Neste artigo é apresentado um aplicativo do jogo de palavras cruzadas para dispositivos smartphones, que tem como objetivo o estímulo à memória visual das palavras, associada à consciência fonológica de crianças disléxicas. O aplicativo também apresenta como objetivo a coleta de dados dos usuários, com o intuito de gerar estatísticas de uso da aplicação, podendo informar, por exemplo, as palavras que possuem uma maior frequência de erros. Informações obtidas por meio de questionários aplicados a fonoaudiólogos apontam para a originalidade e adequação do aplicativo.

\section{Introdução}

Crianças costumam cometer erros durante a fase de aprendizado da escrita. No decorrer do processo de aprendizagem, a criança passa a ter erros específicos e em menor número. Porém, algumas crianças passam a ter uma maior variedade de erros e frequência de erros. Essas dificuldades podem ser sintomas de distúrbios de aprendizado. Entre os distúrbios, está a dislexia [Zorzi e Ciasca, 2008]. 
VI Congresso Brasileiro de Informática na Educação (CBIE 2017)

Anais do XXVIII Simpósio Brasileiro de Informática na Educação (SBIE 2017)

A pessoa disléxica possui dificuldades em associar a representação gráfica das palavras (grafema) com o som que elas representam (fonema). Isso faz com que ocorram problemas na escrita, como troca, omissão, inversão, substituição, junção, acréscimo de letras [Zorzi e Ciasca, 2008]. Também faz com que a leitura muitas vezes seja silabada e que ocorram tentativas de adivinhação da palavra [Zorzi e Ciasca, 2008].

Diante do exposto, pesquisadores investigaram os benefícios das tecnologias da informação e da comunicação (TICs) para promover melhores habilidades de leitura em disléxicos. Em geral, os resultados sugerem que os recursos tecnológicos propiciam aos indivíduos com dislexia maior envolvimento nas atividades de leitura e compreensão de texto [Benmarrakchi et al., 2016; Avelar et al., 2015; Skiada et al., 2014].

Tendo em vista os aspectos observados, esta pesquisa tem como foco o desenvolvimento de uma aplicação que faça uso da atividade palavras cruzadas, com o objetivo de estimular a memória visual das palavras, associada à consciência fonológica de crianças disléxicas. $\mathrm{O}$ aplicativo também tem como objetivo coletar dados dos usuários, gerando, assim, uma estatística de uso, podendo informar, por exemplo, com quais palavras cruzadas as crianças estão tendo mais dificuldade e quais erros estão sendo cometidos com uma maior frequência.

Este artigo é estruturado da seguinte maneira: a Seção 2 apresenta as dificuldades encontradas por crianças disléxicas no aprendizado da leitura e escrita e como o uso de TICs pode ser benéfico ao ensino da leitura e escrita para essas crianças; a Seção 3 apresenta o aplicativo de palavras cruzadas, abordando seus fundamentos, benefícios, aspectos de uso, funcionalidades e ferramentas utilizadas no seu desenvolvimento; a Seção 4 apresenta os resultados de um questionário aplicado a fonoaudiólogos, contemplando uma avaliação do aplicativo proposto; e, por fim, as conclusões e trabalhos futuros são apresentados na Seção 5 .

\section{Ensino da leitura e escrita para disléxicos}

Para o aprendizado da leitura, de acordo com Teles [2004], é necessário que o sujeito possua uma boa consciência fonológica, isto é, o conhecimento consciente de que a linguagem é formada por palavras, as palavras por sílabas, as sílabas por fonemas e que os caracteres do alfabeto representam esses fonemas. Crianças com dislexia, mesmo utilizando palavras, sílabas e fonemas durante a fala, não possuem conhecimento consciente dessas unidades linguísticas [Costa, 2010].

Indivíduos portadores de dislexia tipicamente possuem níveis de leitura e escrita abaixo do esperado. De acordo com Costa [2010], algumas características comuns em pessoas que possuem a desordem são: leitura lenta, sem modulação; confundir algu- 
VI Congresso Brasileiro de Informática na Educação (CBIE 2017)

Anais do XXVIII Simpósio Brasileiro de Informática na Educação (SBIE 2017)

mas letras; sérios erros ortográficos; dificuldades de memória; dificuldades de entender tempo: passado, presente e futuro; escrita desordenada; inversões; omissões; reiterações; e substituição de letras.

Diante do exposto, a associação internacional de dislexia recomenda a utilização de métodos de ensino multissensoriais [International Dyslexia Association, 2016]. Segundo Costa [2010], os métodos de ensino multissensoriais ajudam as crianças a aprender utilizando mais do que um sentido - enfatizam os aspectos cinestésicos da aprendizagem, integrando o ouvir, o ver e o escrever.

Um desafio para indivíduos que apresentam problemas de aprendizado, como a dislexia, de acordo com Zorzi e Ciasca [2008], diz respeito à consolidação das características da escrita regidas por elementos da ortografia. O uso de atividades multissensoriais que estimulem a aprendizagem da ortografia, por meio do reconhecimento visual das palavras associado à consciência fonológica, pode melhorar o desempenho de crianças disléxicas.

As atividades multissensoriais podem ser facilmente aplicadas por meio de aplicativos para dispositivos móveis. O uso de aplicativos para ajudar indivíduos com dislexia pode promover a estimulação via canais visual e auditivo, acelerando o processamento das informações e estimulando o aprendizado da criança [Rahman et al., 2012].

\subsection{TICs aplicadas à dislexia}

Na educação, TIC (Tecnologia da Informação e Comunicação) se refere ao desenvolvimento de tecnologias utilizando componentes de informação e tecnologias de comunicação durante o processo de ensino. De acordo com Benmarrakchi et al. [2016], o estudo de TICs aplicadas à educação vem tendo um crescimento significativo ao longo dos últimos anos. Vários pesquisadores têm provado que o uso de TICs no aprendizado tem resultados positivos, estimulando o interesse dos estudantes. Dentre exemplos de aplicação de TIC em educação, podem ser citados: alfabetoKinect, que é um aplicativo para auxiliar a alfabetização de crianças [Alves et al., 2012]; TAP, um aplicativo para auxílio da aprendizagem da língua estrangeira [Araujo et al., 2012]; uma aplicação de realidade aumentada para auxiliar a aprendizagem de alunos com deficiência intelectual [Santos e Silva Junior, 2016]; e um jogo para ensino de direito do trabalho [Freitas e Adamatti, 2016].

De acordo com pesquisas recentes, o uso de TICs auxilia e reforça o processo de aprendizado de indivíduos disléxicos [Cidrim e Madeiro, 2017]. Em geral, os resultados sugerem que os recursos tecnológicos propiciam aos indivíduos com dislexia maior envolvimento nas atividades de leitura e compreensão de textos [Benmarrakchi et al., 2016; Skiada et al., 2014]. 
VI Congresso Brasileiro de Informática na Educação (CBIE 2017)

Anais do XXVIII Simpósio Brasileiro de Informática na Educação (SBIE 2017)

Com a popularização crescente de dispositivos móveis, mais especificamente os smartphones e os tablets, esses dispositivos tem se tornado a preferência no desenvolvimento de aplicações multissensoriais para crianças com disfunções de aprendizado. Além da popularidade, os dispositivos móveis com os sistemas Android ou IOS possuem uma relativa facilidade quando se trata de desenvolvimento de aplicativos, possuindo uma forte e ampla comunidade, além de ferramentas completas e de fácil aprendizado, como o xcode para o IOS e o Android Studio para o Android.

\section{Aplicação}

Em consonância com os benefícios do uso de atividades multissensoriais no tratamento da dislexia, o aplicativo ora descrito foi desenvolvido para smartphones com o sistema operacional Android, com intuito estimular a memória visual das palavras, associada à consciência fonológica de crianças disléxicas.

O aplicativo foi desenvolvido para a língua portuguesa e conta com uma abordagem multissensorial. Nele a criança terá que resolver exercícios de palavras cruzadas com foco em sílabas, com recurso de pista acústica da palavra. Todos os aspectos visuais da aplicação levaram em consideração as características dos sujeitos alvos, ou seja, das crianças disléxicas. O aplicativo também tem como objetivo coletar dados dos usuários, gerando, assim, uma estatística de uso, podendo informar, por exemplo, com que palavras as crianças têm mais dificuldade e que erros são cometidos com uma maior frequência.

Um aspecto a destacar é a originalidade do aplicativo. De acordo com o conhecimento dos autores, não há, na literatura e em lojas virtuais, aplicativo de jogo de palavras cruzadas que possua uma proposta similar à do aplicativo desenvolvido. Dentre os componentes de originalidade, destacam-se: uso de sílabas, visual adequado ao usuário disléxico, uso de pista sonora.

\subsection{Aspectos de usabilidade}

Os aspectos de usabilidade da aplicação são resultantes de um conjunto de recomendações e diretrizes realizadas por uma fonoaudióloga especialista em dislexia e doutoranda em Ciências da Linguagem pela Universidade Católica de Pernambuco (UNICAP). A interface foi desenvolvida para ser adequada ao usuário disléxico, assim facilitando o processo de leitura e estimulando o usuário a utilizar a aplicação.

A fonte escolhida para a aplicação foi a Open Dyslexic. Trata-se de uma fonte de código aberto, com objetivo de aumentar a legibilidade dos textos para disléxicos. A fonte é continuamente atualizada por feedback de portadores de dislexia [OPENDYSLEXIC, 2017]. 
VI Congresso Brasileiro de Informática na Educação (CBIE 2017)

Anais do XXVIII Simpósio Brasileiro de Informática na Educação (SBIE 2017)

Na Figura 1 pode ser observada a fonte de texto Open Dyslexic e outras fontes mais populares, como a verdana e a Times. É possível observar que na fonte Open dyslexic as letras possuem uma base com um desenho largo, para que o leitor rapidamente identifique qual parte da letra está para baixo, auxiliando no reconhecimento correto da letra [OPENDYSLEXIC, 2017].

As cores da aplicação foram escolhidas de maneira a evitar o uso de letra preta e fundo branco. De acordo com Avelar et al. [2015], o uso de letra preta a fundo branco faz com que os indivíduos disléxicos, após um tempo de uso da aplicação, comecem a ver "padrões visuais" e "letras se movendo" nos textos.

Todos os sons de efeitos utilizados na aplicação foram retirados de uma base de som gratuita da Internet, com licenças que permitem a utilização dos mesmos. Para os sons das palavras, foi utilizada a biblioteca de conversão de texto para fala do Android. A biblioteca permite que o texto escrito seja convertido em palavras faladas. Ela está disponível em todos os dispositivos Android, e não é necessário que o usuário instale outra aplicação para que a mesma funcione.

\begin{tabular}{c|cccc} 
Gill Sans & rn m & MW & dpqb & IIlijJ \\
Verdana & rn m & MW & dpqb & I1IijJ \\
OpenDyslexic & rn m & MW & dpqb & I1IijJ \\
Times & rn m & MW & dpqb & 11 IijJ \\
Helvetica & rn m & MW & dpqb & I1lijJ
\end{tabular}

Figura 1. Fonte de texto Open Dyslexic e fontes usualmente disponíveis em programas de edição de texto [OPENDYSLEXIC, 2017].

\subsection{Funcionalidade}

A criança pode fazer uso do aplicativo estando só, entretanto, no primeiro uso é recomendado que o funcionamento do aplicativo seja explicado à criança por um adulto ou pelo fonoaudiólogo. Com o aplicativo, é esperado que a criança se divirta e que exercite sua memória visual associada à consciência fonológica.

Ao iniciar o aplicativo, o usuário irá se deparar com a tela inicial. Nessa tela ele poderá clicar em jogar, sendo então direcionado para a tela de seleção de níveis, ou poderá clicar em configurar, sendo direcionado para a tela de configuração do aplicativo. 
VI Congresso Brasileiro de Informática na Educação (CBIE 2017)

Anais do XXVIII Simpósio Brasileiro de Informática na Educação (SBIE 2017)

Na Figura 2 é possível observar a tela de seleção de níveis. As palavras cruzadas desenvolvidas para o aplicativo foram divididas em níveis. Os critérios para definições dos níveis foram: quantidade de palavras - quanto maior o número de palavras, maior é o nível de dificuldade; complexidade das sílabas - sílabas complexas têm um maior nível de dificuldade que sílabas simples; quantidade de sílabas por palavra - palavras que possuem um número maior de sílabas possuem um nível maior de dificuldade.

Após escolha do nível, o usuário é direcionado para a tela da palavra cruzada. $\mathrm{O}$ aplicativo ora descrito, diferentemente dos jogos de palavras cruzadas comuns, separa as palavras por sílabas em vez de separa-las por letras. As pistas do aplicativo fazem uso de recurso sonoro. Ao clicar nos ícones de som, a criança poderá ouvir o som da palavra que ela deve preencher na linha ou coluna.

O usuário, para completar a palavra cruzada, terá de preencher os espaços com as sílabas que ficam na parte inferior da tela. Para isto, ele terá de clicar e arrastar as sílabas para as posições até formar as palavras corretamente. Caso o usuário posicione uma sílaba de forma incorreta, o aplicativo dá um feedback de erro de acordo com o que foi estabelecido na tela de configurações, e a sílaba deve ser removida da posição escolhida clicando nela novamente. Caso o usuário posicione uma sílaba corretamente, ele não poderá mais removê-la. Ao preencher todas as sílabas corretamente, o aplicativo exibe uma mensagem parabenizando a criança, e ela será direcionada de volta para a tela de seleção de níveis. Na Figura 2 é possível observar telas da palavra cruzada: a parte cinza é onde ficam as sílabas a serem escolhidas; os blocos azuis com símbolo de som são botões que, quando pressionados, emitem o som da palavra. Na tela mais a direita da Figura 2 é possível observar o feedback visual de erro.
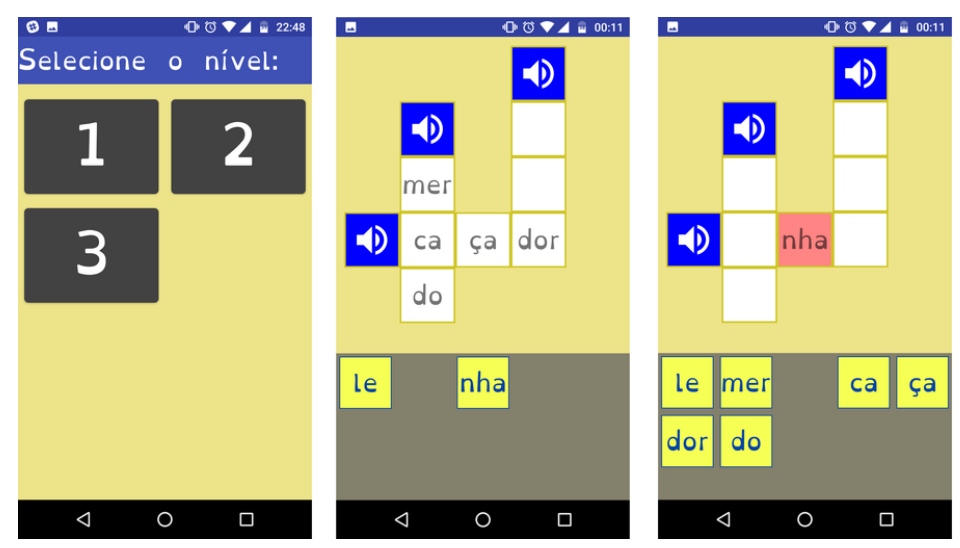

Figura 2. Telas palavras cruzadas.

Nas configurações do aplicativo, o usuário pode configurar o feedback de erro. A aplicação possui três tipos de feedback de erro que podem ser desativados ou ativados. Os feedbacks são: sonoro, em que, a acriança poderá ouvir um barulho, indicando que ela 
VI Congresso Brasileiro de Informática na Educação (CBIE 2017)

Anais do XXVIII Simpósio Brasileiro de Informática na Educação (SBIE 2017)

escolheu a sílaba incorreta; tátil, em que, o aplicativo faz o dispositivo vibrar para alertar do erro cometido; visual, em que, após a sílaba ser posicionada em um local errado, a cor de fundo do texto irá mudar para vermelho, indicando que a sílaba está incorreta. Essas opções permitem que o responsável ou fonoaudiólogo da criança configure o aplicativo, de maneira que o feedback de erro seja mais adequado à criança. $\mathrm{O}$ alvo é evitar que o erro desestimule o uso do aplicativo.

\subsection{Relatório de uso}

O relatório de uso da aplicação permite que o orientador das crianças descubra quais palavras possuem uma maior ocorrência de erros. Permite também a identificação da sílaba da palavra com maior ocorrência de erros.

A ferramenta de análise estatística utilizada no desenvolvimento da aplicação foi a Flurry analytics, a qual faz coleta e análise de aplicações para dispositivos móveis que possuem os sistemas operacionais Android ou IOS. A ferramenta conta com mais de 250.000 desenvolvedores e com mais de 940.000 aplicativos registrados [Yahoo Developer Network, 2017].

Para a escolha da ferramenta, foram considerados os seguintes critérios: documentação completa; facilidade de uso; custo de utilização; reconhecimento pela comunidade; possibilidade de exportação dos dados; disponibilidade de dados em tempo real.

Durante a execução da palavra cruzada, a aplicação armazena todos os erros cometidos em uma sessão, para serem submetidos para o servidor ao fim da sessão. Cada sessão inicia quando uma palavra cruzada é iniciada e pode ser finalizada em cenários diferentes: quando o usuário fecha a aplicação durante a execução da palavra cruzada ou quando ele completa a palavra cruzada. Para a coleta dos erros, foi utilizada a funcionalidade de eventos personalizados disponibilizada pelo Flurry Analytics.

Na Figura 3 é possível ver o painel que apresenta os eventos de erro submetidos ao servidor em ordem decrescente pelo número de ocorrências do mesmo. Neste painel é possível identificar os seguintes dados: em qual palavra está ocorrendo o maior número de erros; a quantidade média de erros que ocorreram na palavra por sessão; e a quantidade de média de erros que ocorreram na palavra por dia.

O painel de visualização para análise dos parâmetros dos eventos pode ser visto na Figura 4. Neste painel é possível analisar os erros de uma determinada palavra, como por exemplo qual sílaba da palavra possui o maior número de erros ou quais sílabas estão sendo utilizadas de maneira errada para formar a palavra com maior frequência. Estes dados podem, por exemplo, auxiliar o fonoaudiólogo na hora de elaborar exercícios para a intervenção nas crianças disléxicas. 
VI Congresso Brasileiro de Informática na Educação (CBIE 2017)

Anais do XXVIII Simpósio Brasileiro de Informática na Educação (SBIE 2017)

\begin{tabular}{|c|c|c|c|c|c|c|c|}
\hline Event Name & & & App Name & $\begin{array}{c}\text { Total Event } \\
\text { Occurrences v }\end{array}$ & $\begin{array}{r}\text { Avg Events per } \\
\text { Session }\end{array}$ & $\begin{array}{r}\text { Event Occurrences } \\
\text { Daily Avg }\end{array}$ & $\begin{array}{r}\text { Unique Event } \\
\text { Devices Daily Avg }\end{array}$ \\
\hline \multirow[t]{2}{*}{ Justiça } & 国 & 四 & 해 Dyslexia & 14 & 0.5 & 0.5 & 1 \\
\hline & & & Crosswords & & & & \\
\hline \multirow[t]{2}{*}{ Caçador } & 国 & 四 & Dyslexia & 7 & 0.3 & 0.2 & 1 \\
\hline & & & Crosswords & & & & \\
\hline \multirow[t]{2}{*}{ Travesseiro } & 国 & 四 & - Dyslexia & 6 & 0.2 & 0.2 & 1 \\
\hline & & & Crosswords & & & & \\
\hline \multirow[t]{2}{*}{ Vestido } & 国 & 四 & की Dyslexia & 6 & 0.2 & 0.2 & 1 \\
\hline & & & Crosswords & & & & \\
\hline \multirow[t]{2}{*}{ Mercado } & 国 & 四 & Dyslexia & 4 & 0.1 & 0.1 & 1 \\
\hline & & & Crosswords & & & & \\
\hline
\end{tabular}

Figura 3. Painel de resumo dos eventos.

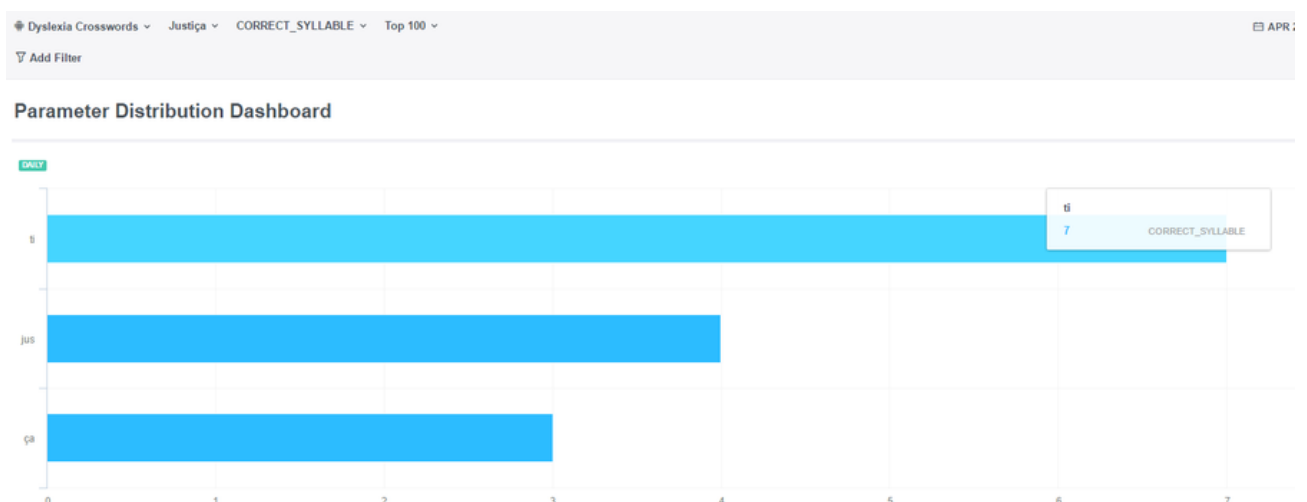

Figura 4. Painel de distribuição dos parâmetros.

\section{Resultados}

Nesta seção são apresentados os resultados obtidos por meio da aplicação de um questionário a nove fonoaudiólogos. Esses profissionais foram convidados a responder perguntas (Tabela 1) e apresentar as justificativas correspondentes.

Conforme se observa na Tabela 1, os resultados apontam para uma boa aceitação da aplicação. Observa-se, segundo os profissionais que responderam o questionário, que a aplicação possui um uso intuitivo. Todos os fonoaudiólogos responderam que utilizariam o aplicativo como parte das atividades a serem aplicadas a usuários disléxicos, e justificaram essa reposta com base na diversão proporcionada à criança pela aplicação e em virtude da atração que crianças disléxicas têm por tecnologia. Nenhum dos entrevistados declarou conhecer um aplicativo com proposta semelhante à apresentada. 
VI Congresso Brasileiro de Informática na Educação (CBIE 2017)

Anais do XXVIII Simpósio Brasileiro de Informática na Educação (SBIE 2017)

As estatísticas de uso da aplicação despertaram interesse da maior parte dos profissionais: seis deles responderam ter interesse e enfatizaram que o acesso às palavras erradas ajuda a construir novas atividades, enquanto três declararam não ter interesse, em virtude da falta de tempo para análise dos dados.

Tabela 1. Pergunta x Resultado da pesquisa.

\begin{tabular}{|l|c|c|}
\hline \multicolumn{1}{|c|}{ Pergunta } & Sim & Não \\
\hline O uso do aplicativo é intuitivo? & 9 & 0 \\
\hline $\begin{array}{l}\text { Você identifica no aplicativo elementos que dificultam o uso para } \\
\text { crianças disléxicas? }\end{array}$ & 0 & 9 \\
\hline $\begin{array}{l}\text { Você considera a pista acústica das palavras em conjunto com o uso } \\
\text { de sílabas mais intuitivo para crianças disléxicas que o uso de letras } \\
\text { em palavras cruzadas convencionais? }\end{array}$ & 9 & 0 \\
\hline $\begin{array}{l}\text { Você utilizaria este aplicativo como parte das atividades a serem } \\
\text { aplicadas a uma criança disléxica? Justifique. }\end{array}$ & 9 & 0 \\
\hline $\begin{array}{l}\text { Você teria interesse em ter acesso às estatísticas de uso do } \\
\text { aplicativo, como, por exemplo, a frequência dos erros cometidos } \\
\text { pela criança disléxica? }\end{array}$ & 6 & 3 \\
\hline $\begin{array}{l}\text { Você conhece aplicativo(s) com proposta semelhante? Em caso } \\
\text { afirmativo, informe qual(is)? }\end{array}$ & 0 & 9 \\
\hline
\end{tabular}

\section{Conclusão}

Neste trabalho, foi apresentado um aplicativo de palavras cruzadas para a plataforma Android, com o objetivo de estimular a memória visual de crianças disléxicas, associada à consciência fonológica. O aplicativo foi apresentado a profissionais da área de fonoaudiologia, que subsequentemente foram convidados a responder um questionário.

O aplicativo se mostra adequado ao que se propõe. Os fonoaudiólogos avaliados mostraram interesse pela aplicação e pelos dados de utilização gerados pela mesma. Destaca-se a originalidade da aplicação: nenhum dos fonoaudiólogos declar conhecer algum aplicativo com proposta semelhante.

Como trabalhos futuros, a aplicação será submetida a uma avaliação formal por educadores e mais profissionais da área de fonoaudiologia, para que seja aplicado a crianças disléxicas. Também devem ser realizadas melhorias na interface gráfica da aplicação para torná-la ainda mais atrativa aos usuários disléxicos.

\section{Referências}

Alves, R. S., de Araujo, J. O. A., e Madeiro, F. (2012). AlfabetoKinect: Um aplicativo para auxiliar na alfabetização de crianças com o uso do Kinect. In Anais do $23^{\circ}$ Simpósio 
VI Congresso Brasileiro de Informática na Educação (CBIE 2017)

Anais do XXVIII Simpósio Brasileiro de Informática na Educação (SBIE 2017)

Brasileiro de Informática na Educação.

Araujo, J. O. A., Madeiro, F., de Moura, A. L. B., e de S. Alves, R. (2012). TAP: Um aplicativo para auxílio à aprendizagem de língua estrangeira. In Anais do $23^{\circ}$ Simpósio Brasileiro de Informática na Educação.

Avelar, L. O., Rezende, G. C., e Freire, A. P. (2015). WebHelpDyslexia: A browser extension to adapt web content for people with dyslexia. Procedia Computer Science, 67, $150-159$.

Benmarrakchi, F., Kafi, J. E., Elhore, A., e Haie, S. (2016). Exploring the use of the ICT in supporting dyslexic students' preferred learning styles : A preliminary evaluation. Education and Information Technologies, pages 1 - 19.

Cidrim, L. e Madeiro, F. (2017). Tecnologias da informação e da comunicação (TIC) aplicadas à dislexia: revisão de literatura. Rev CEFAC, 19(1), 99 - 108.

Costa, A. L. A. (2010). Distúrbios de leitura e escrita: as dificuldades na aprendizagem da leitura com pessoas disléxicas. Biblionline, pages $99-108$.

Freitas, V. C. B. e Adamatti, D. (2016). Educa Direito: um jogo sério para o ensino de direito do trabalho. Revista Brasileira de Informática na Educação, 24(3).

International Dyslexia Association (2016). Multisensory structured languade teaching. https://dyslexiaida.org/multisensory-structured-language-teaching/.

OPENDYSLEXIC (2017). About. https://opendyslexic.org/about-2/.

Rahman, F. A., Mokhtar, F., Alias, N. A., e Saleh, R. (2012). Multimedia elements as instructions for dyslexic children. International Journal of Education and Information Technologies, 6(2), $193-200$.

Santos, W. O. e Silva Junior, C. G. (2016). Realidade aumentada e gamificação na educação: uma aplicação para auxiliar no processo de aprendizagem de alunos com deficiência intelectual. Revista Brasileira de Informática na Educação, 24(1).

Skiada, R., Soroniati, E., Gardeli, A., e Zissis, D. (2014). EasyLexia 2.0: Redesigning our mobile application for children with learning difficulties. Themes in Science \& Technology Education, 7, 119 - 135.

Teles, P. (2004). Dislexia: Como identificar? Como intervir? Rev Port Clin Geral, 20, 713 -730 .

Yahoo Developer Network (2017). Flurry Analytics. https://developer.yahoo.com/analytics/.

Zorzi, J. L. e Ciasca, S. M. (2008). Caracterização dos erros ortográficos em crianças com transtornos de aprendizagem. Rev CEFAC, 10(3), $321-331$. 